\title{
Detección de anticuerpos de Brucella spp. en mataderos del estado Bolívar y Soledad, municipio Independencia, estado Anzoátegui, Venezuela, 2006
}

\author{
Screening of antibodies to Brucella spp. in slaughterhouses of the Bolívar state and \\ Soledad, Independencia Municipality, Anzoategui state, Venezuela, 2006
}

\author{
Julman Rosiris Cermeño* y Ricardo De Grazia. \\ Departamento de Parasitología y Microbiología, Escuela de Ciencias de la Salud "Dr. Francisco Battistini Casalta", \\ Universidad de Oriente, Núcleo Bolívar. Ciudad Bolívar. Venezuela. *Correo electrónico: jcerme30@gmail.com
}

\begin{abstract}
RESUMEN
El objetivo de este estudio fue determinar la seroprevalencia humana y bovina de anticuerpos contra Brucella spp. en mataderos del estado Bolívar y Soledad, municipio Independencia, estado Anzoátegui, Venezuela. Para ello, se realizó un estudio prospectivo y transversal. Previo permiso por escrito de autoridades competentes y consentimiento informado de los trabajadores, se seleccionaron 7 mataderos. Se registraron datos de interés epidemiológico y clínico. Se extrajo sangre por venopunción de personas y bovinos. La demostración de anticuerpos contra Brucella spp. se realizó mediante la prueba DRG $®$ Brucella IgM ELISA en humanos y ELISA competitiva, Brucella-Ab C-ELISA de SVANOVIR®, en animales. Se evaluaron 462 sueros: 159 de trabajadores y 303 de bovinos. La prevalencia de infección en humanos fue 5,6 \% y de bovinos $11,2 \%$; el mayor porcentaje se obtuvo en el grupo de los trabajadores con edades entre 21 y 30 años $(a)(P=0,15)$ y de sexo masculino $(P=0,34)$. Siendo más frecuente en el personal que tenía menos de 10 a con riesgo ocupacional $(66,6 \%)$; correspondiendo a la ocupación de matarife y despostador el mayor número de casos. El 55,6 \% de los trabajadores consumían lácteos sin pasteurizar. Las manifestaciones clínicas fueron inespecíficas: artralgias, cefalea y sudoración nocturna $(P>0,05)$. Se demuestra que la mayoría de trabajadores de los mataderos evaluados del estado Bolívar y Soledad, municipio Independencia del estado Anzoátegui, están expuestos a múltiples factores de riesgo para contraer brucelosis, sin embargo, la seroprevalencia de anticuerpos de Brucella spp. en ellos fue baja (5,6 \%) aunque elevada entre los bovinos estudiados $(11,2 \%)$.
\end{abstract}

Palabras clave: Bovinos; brucelosis; seroprevalencia

\begin{abstract}
The aim of this study was to determine the seroprevalence of human and bovine antibodies to Brucella spp in Bolivar State slaughterhouses and Soledad, Independencia Municipality, Anzoategui State, Venezuela. To this end, it was conducted to a prospective, cross study. Prior written consent of workers and permission of authorities, seven slaughterhouses were selected. Data were recorded epidemiological and clinical interest. Blood was drawn by venipuncture from subjects and cattle. Demonstration of Brucella spp. antibodies was performed IgM ELISA DRG® Brucella in humans and competitive ELISA, Brucella Ab C-ELISA SVANOVIR ${ }^{\circledR}$, in animals. Four hundred sixty two sera were evaluated: 159 workers and 303 cattle. The prevalence of human infection was $5.6 \%$ and $11.2 \%$ of cattle; the highest percentage was obtained in the group of workers aged between 21 and 30 years $(y r)$ old $(P=0.15)$ and male $(P=0.34)$. Being more common in people who were younger than $10 \mathrm{yr}$ with occupational risk $(66.6 \%)$, corresponding to the occupation of butcher and stripper the largest number of cases. The $55.6 \%$ of workers consumed unpasteurized dairy. Clinical manifestations were nonspecific: arthralgia, headache and night sweats $(P>0.05)$. It was showed that most of the studied slaughterhouse workers in Bolivar State and Soledad, Independencia Municipality of Anzoátegui State, are exposed to multiple risk factors for acquiring brucellosis, however, the seroprevalence of antibodies to Brucella spp. in them was low $(5.6 \%)$ but higher among the studied cattle (11.2\%).
\end{abstract}

Key words: Cattle; brucellosis; seroprevalence 


\section{INTRODUCCIÓN}

La brucelosis es una de las zoonosis desatendida más común en todo el mundo; transmitida al hombre por diversos animales: ganado bovino (Bos taurus), ovino (Ovis orientalis aries), caprino (Capra aegagrus hircus), porcino (Sus scrofa domesticus), camellos (Camelus), búfalos (Bubalus bubalis), entre otros [15]. Afecta tanto a los países desarrollados y en desarrollo [23, 26]. La enfermedad es frecuente en la región mediterránea, oeste de Asia, África y América Latina [2, 15, 26].

La infección por Brucella spp. se presenta como consecuencia de la exposición, contacto con animales infectados (tejidos, sangre, orina, heces, secreciones vaginales), y/o algunos de sus derivados (leche y de productos lácteos no pasteurizados), o por la transmisión aérea; en consecuencia los más afectados, son los trabajadores agropecuarios y de mataderos, ya que tienen un alto índice de cortaduras, exposición a la sangre y/o linfa de animales recién muertos, por lo que se ha considerado como una enfermedad ocupacional [8, 15, 24].

Las especies más comunes que afectan al ganado y a los humanos son: $B$. abortus, $B$. suis y $B$. melitensis, que infectan preferencialmente (pero no exclusivamente) al ganado, cerdos y pequeños rumiantes, respectivamente. Otras especies de Brucella incluyen $B$. ovis (ovejas), B. canis (perros), B. neotomae (roedores), B. microti (topillos), B. pinnipedialis (pinnípedos), B. ceti (cetáceos), $B$. papionis (babuinos), B. vulpis (zorros) y B. inopinata [36].

En los países en desarrollo donde la brucelosis es endémica, conocer los datos de referencia sobre la prevalencia de Brucella spp. empleando las instalaciones de los mataderos, son importantes [22]; ya que se han asociado las exposiciones ocupacionales a la infección con este microorganismo, particularmente entre los trabajadores [12].

En Venezuela, como en muchos países de Latinoamérica, la brucelosis es una enfermedad que causa importantes pérdidas económicas, especialmente en el ganado bovino, y constituye un grave problema de salud pública ya que representa una amenaza para quienes producen, distribuyen y consumen productos lácteos sin pasteurizar [7]. La brucelosis bovina en Venezuela tiene índices bajos según la cifras oficiales, están entre 0,8 a $14 \%$ [35]. Actualmente la vigilancia epidemiológica de brucelosis bovina en el país consiste en la recolección de la información de las actividades que ejecutan los médicos veterinarios en ejercicio libre, las cuales se procesan a nivel de las oficinas sanitarias locales [28, 35]. La especie predominante en el país es la $B$. abortus, tanto en humanos como en animales, produciendo en la población bovina altas tasas de abortos en hembras e infertilidad en machos, siendo transmisible a humanos expuestos ocupacionalmente [35].

En la actualidad se requieren de estudios clínicos y epidemiológicos (humano y animal) en los diferentes Estados del país para conocer la verdadera prevalencia e incidencia de la brucelosis y su impacto socio-económico. En el estado Bolívar, se desconoce la situación actual de la brucelosis humana y bovina; por ello, se planteó el presente estudio con la finalidad de determinar la seroprevalencia de Brucella spp. en los trabajadores de los mataderos y en el ganado bovino del estado Bolívar y Soledad, municipio Independencia del Edo. Anzoátegui, cuyo matadero suplía las funciones del municipio Angostura del Orinoco, del estado Bolívar, Venezuela.

\section{MATERIALES Y MÉTODOS}

Se realizó un estudio descriptivo, transversal realizado en forma prospectiva.

El estado Bolívar tiene 11 Municipios y posee 9 mataderos en total, ubicados en los municipios: Angostura del Orinoco (antes Heres), Caroní, Piar, Padre Pedro Chien, Cedeño, Sifontes, Roscio, El Callao y Gran Sabana. El matadero del municipio Angostura del Orinoco no estaba en funcionamiento en el momento del estudio, por tal motivo el Matadero Municipal de Soledad, municipio Independencia del estado Anzoátegui, suplía el área de influencia del municipio Angostura del Orinoco del estado Bolívar (100 \%), motivo por el cual se incluyó este último matadero.

Se seleccionaron 7 mataderos, previo permiso de los dueños del ganado y autoridades competentes; además, del consentimiento voluntario de los trabajadores para realizar el estudio; se respetaron los principios éticos para la investigación médica en seres humanos, siguiendo los lineamientos de la Declaración de Helsinki [3]. Este estudio fue aprobado por la Comisión de Tesis de Grado de la Universidad de Oriente, Venezuela, quien revisa los aspectos éticos y metodológicos de la investigación.

\section{Población y muestra humana}

De un total de 209 trabajadores, se evaluaron 159 quienes aceptaron participar en forma voluntaria en el estudio, y que constituían el $76,1 \%$ del total de los trabajadores de los mataderos incluidos: $28(80 \%)$ trabajadores del matadero municipal de Soledad; $36(55,4 \%)$ trabajadores del matadero Maloca C.A., San Félix; 28 (80\%) trabajadores del matadero municipal de Upata; 18 (90\%) trabajadores de la sala de matanza municipal de El Callao; $20(90,9 \%)$ trabajadores de la sala de matanza municipal de Guasipati; 12 (80\%) trabajadores de la sala de matanza municipal de Tumeremo; y 17 (100\%) trabajadores de la sala de matanza municipal de El Palmar.

Los datos de identificación personal, epidemiológicos y manifestaciones clínicas fueron recolectados de manera independiente en una ficha individual diseñada para tal fin.

De cada individuo se obtuvo una muestra sanguínea de la vena cubital (5 - 6 mililitros $(\mathrm{mL})$ en tubos estériles al vacío (Vacutainer $\left.{ }^{\circledR}\right)$. Media hora después de su obtención, las muestras fueron centrifugadas (Centrifuge Digisystem Laboratory Instrument, Inc; Modelo DSCI58T, Taiwan) a $3.500 \mathrm{G}$ x $10 \mathrm{~min}$, obtenido el suero y fraccionándolo en alícuotas de $1 \mathrm{~mL}$ y éstos fueron conservados a $-20^{\circ} \mathrm{C}$ (Thermo Scientific ${ }^{\mathrm{TM}}$, GP Series; USA) hasta su procesamiento.

\section{Población y muestra bovina}

En todo el estado Bolívar, para el momento del estudio, se sacrificaban aproximadamente de 9.000 a 9.100 cabezas de ganado bovino en un mes, de los cuales 3.000 se benefician en el matadero de Soledad, municipio Independencia, que suplía las funciones del matadero del municipio Angostura del Orinoco; 3.500, en el matadero Maloca C.A. de San Félix; 850 en el Matadero Municipal de Upata; 300 en la Sala de Matanza Municipal de El Callao; 580 en la Sala de Matanza Municipal de Guasipati; 450 en la Sala de Matanza Municipal de Tumeremo y 300 en la Sala de Matanza Municipal de El Palmar. 
De un total de 303 bovinos seleccionados en un día (d) de matanza (100\%), se estudiaron 115 bovinos (37,9\%) en el matadero Maloca C.A. de San Félix; 97 (32,0 \%) en el Matadero Municipal de Soledad, municipio Independencia del Edo. Anzoátegui; 30 (9,9\%) en el Matadero Municipal de Upata; $19(6,3 \%)$ en la Sala de Matanza Municipal de Guasipati; 17 (5,6 \%) en la Sala de Matanza Municipal de El Palmar; 15 (5,0 \%) en la Sala de Matanza Municipal de Tumeremo y 10 (3,3\%) en la Sala de Matanza Municipal de El Callao.

En cada uno de los mataderos se tomó una muestra de sangre venosa yugular $(8-10 \mathrm{~mL})$ en tubos sin anticoagulante (Vacutainer $^{\circledR}$ ), de la totalidad de los bovinos que fueron llevados para sacrificio al matadero, en un d de matanza escogido al azar, sin historia clínica confirmada de brucelosis. Se separó el suero y se congeló a $-40^{\circ} \mathrm{C}$ (Thermo Scientific ${ }^{\top M}$, TSX, USA) hasta el momento de la realización de las diferentes pruebas. Todas las muestras fueron conservadas hasta su procesamiento en el Departamento de Parasitología y Microbiología de la Universidad de Oriente, Núcleo Bolívar.

La recolección de las muestras, en los bovinos, de los diferentes mataderos se realizó en el período comprendido desde enero a septiembre del año 2006.

\section{Pruebas serológicas}

Se empleó la prueba inmunoenzimática indirecta para la detección de anticuerpos IgM frente a Brucella spp. en suero humano, DRG ${ }^{\circledR}$ Brucella IgM ELISA (EIA-3456), DRG International, Inc USA [11]. La detección de anticuerpos contra Brucella spp. en los bovinos se realizó mediante la prueba de ELISA competitiva, Brucella-Ab C-ELISA de SVANOVIR ${ }^{\circledR}$, ésta se utiliza para la detección de los anticuerpos de Brucella spp. en suero, de manera confiable y rápida, discriminando entre los animales infectados y vacunados, además de ser una prueba multiespecie (sensibilidad del 98,0 \% y especificidad el 99,7\%) [32]. Estas pruebas fueron realizadas e interpretadas siguiendo las recomendaciones del fabricante.

\section{Análisis estadístico}

Se realizó estadística descriptiva. Las variables cualitativas se expresaron indicando las frecuencias absolutas y la proporción de cada una de las categorías, y las cuantitativas con sus medias y desviaciones estándar. Se empleó la prueba de Ji al cuadrado $\left(\mathrm{Ji}^{2}\right)$ y el Test Exacto de Fisher para comparar las variables cualitativas. El nivel de significación utilizado fue $P \leq 0,05$. Se utilizó el paquete estadístico SPSS versión 21,0 para ordenador IBM.

\section{RESULTADOS Y DISCUSIÓN}

Se procesaron un total de 462 muestras de sueros, de las cuales 159 fueron obtenidas de los trabajadores y 303 de los bovinos. Los trabajadores evaluados tenían una edad media de 36,5 \pm 12,3 años (a), con un rango entre 14 y 71 a, siendo predominante el grupo etario entre 31 - 40 a de edad y el género masculino $(n=152 ; 95,6 \%)$.

Los anticuerpos específicos contra Brucella spp. fueron demostrados en 9 trabajadores, lo que representó una prevalencia del 5,6\%. El mayor porcentaje de seropositividad se evidenció en el grupo entre 21 a 30 a de edad ( $n=5 ; 3,1 \%)$, predominando el sexo masculino $(n=8 ; 5,1 \%)(P=0,34)$. Sólo una mujer $(0,6 \%)$ resultó positiva; hay que destacar que ella era veterinaria e inspectora de salud pública de las salas de matanzas del estado Bolívar $(P>0,05)$.

La mayor prevalencia de anticuerpos contra Brucella spp. se encontró en población joven con edades comprendidas entre 21 a 30 a, coincidiendo con algunos estudios realizados en México [22]. A diferencia de otros realizados en Almería, España, donde predomina en el grupo comprendido entre 40 y 49 a [16]; esto evidencia una diferencia en la mayoría de las series y probablemente se deba en gran parte al período de mayor actividad laboral en cada región. La mayoría de los trabajadores eran del sexo masculino, lo que coincide con otros investigadores [16, 22, 29] y guarda relación con la ocupación que los expone a esta enfermedad.

Este estudio demostró una baja seroprevalencia de infección por Brucella spp. (5,6 \%) en los trabajadores de los mataderos evaluados, coincidiendo con otra investigación realizada en el país [5]. Prevalencias elevadas han sido registradas en otras latitudes como Irán $(29,8 \%)$ [12], Pakistán (21,7\%) [24], México $(18,1 \%)$ [8], India (9,9 \%) [2], Kyrgyzstan (8,8 \%) [6] y Malasia (5,4\%) [19]. Cifras inferiores de seroprevalencia en humanos se han descrito en Brasil (0,6 \%) [17] y Bangladesh (4,4 \%) [27].

Hubo predominio de la infección por Brucella spp. en los trabajadores del matadero de San Félix $(n=4 ; 2,5 \%)$ seguido del de Tumeremo $(n=3 ; 1,8 \%)(P>0,05)$, lo que implica que no hay diferencias entre los mataderos, aunque la frecuencia de uno sea mayor que el del otro (TABLA I).

\section{TABLA I}

Anticuerpos específicos contra Brucella spp. en trabajadores de las salas de matanzas según grupo etario, sexo y ubicación de mataderos en el Edo. Bolívar y Soledad municipio Independencia (Edo. Anzoátegui)

\begin{tabular}{ccrr}
\hline & \multicolumn{3}{c}{ Anticuerpos contra Brucella spp. } \\
Variables & Negativo (\%) & Positivo $(\%)$ & Total $(\%)$ \\
\hline EDAD (años)* & & & \\
$10-20$ & $0(0,0)$ & $18(11,3)$ & $18(11,3)$ \\
$21-30$ & $5(3,1)$ & $33(20,8)$ & $38(23,9)$ \\
$31-40$ & $1(0,6)$ & $44(27,7)$ & $45(28,3)$ \\
$41-50$ & $3(1,9)$ & $31(19,5)$ & $34(21,3)$ \\
$51-60$ & $0(0,0)$ & $21(13,2)$ & $21(13,2)$ \\
$>60$ & $0(0,0)$ & $3(1,9)$ & $3(1,9)$ \\
Total & $9(5,6)$ & $150(94,4)$ & $159(100,0)$ \\
\hline SEXO** & & & \\
Masculino & $8(5,1)$ & $144(90,5)$ & $152(95,6)$ \\
Femenino & $1(0,6)$ & $6(3,8)$ & $7(4,4)$ \\
\hline MATADEROS & & & \\
San Félix & $4(2,5)$ & $32(20,1)$ & $36(22,6)$ \\
Tumeremo & $3(1,8)$ & $9(5,6)$ & $12(7,5)$ \\
Upata & $2(1,3)$ & $26(16,3)$ & $28(17,6)$ \\
Soledad & $0(0,0)$ & $28(17,6)$ & $28(17,6)$ \\
Guasipati & $0(0,0)$ & $20(12,6)$ & $20(12,6)$ \\
El Callao & $0(0,0)$ & $18(11,3)$ & $18(11,3)$ \\
El Palmar & $0(0,0)$ & $17(10,7)$ & $17(10,7)$ \\
\hline
\end{tabular}

${ }^{*} \mathrm{j}^{2}=8,021: 5^{\circ} \mathrm{L} ; \mathrm{P}=0,155 ; * \star \mathrm{j}^{2}=1,020: 1^{\circ} \mathrm{L} ; \mathrm{P}=0,340$ 
Se registró mayor frecuencia de infección por Brucella spp. en el personal que tenía menos de 10 a con riesgo ocupacional $(n=6$; $3,8 \%$ ). El cargo de matarife mostró el mayor número de casos seropositivos $(n=5 ; 3,1 \%)$, seguido del de despostador $(n=2$; $1,2 \%)$ (TABLA II).

La frecuencia de seropositividad a Brucella spp. depende en gran medida del tiempo de exposición al riesgo y del contacto directo con los animales o sus derivados [16, 27], así como fue demostrado en el presente estudio con la ocupación de matarife y despostador. Lo anterior, también es válido con la ocupación previa, y se demuestra porque la mayoría de los casos seropositivos fueron aquellos trabajadores que tenían el

Tabla II

Prevalencia de anticuerpos específicos contra Brucella spp. según años de trabajo, ocupación actual y previa en el Edo. Bolívar y Soledad municipio Independencia (Edo. Anzoátegui)

\section{Anticuerpos contra Brucella spp.}

Variables

Positivos Negativos

Total

(\%)

(\%)

(\%)

Tiempo (años) *

\begin{tabular}{cccc}
$\leq 10$ & $6(3,8)$ & $96(60,4)$ & $102(64,2)$ \\
$>10$ & $3(1,8)$ & $54(33,9)$ & $57(35,8)$ \\
Total & $9(5,6)$ & $150(94,4)$ & $159(100,0)$ \\
\hline
\end{tabular}

Ocupación actual

$\begin{array}{lrrr}\text { Matarife } & 5(3,1) & 68(42,8) & 73(45,9) \\ \begin{array}{l}\text { Despostador } \\ \text { Inspector de } \\ \text { salud pública }\end{array} & 2(1,2) & 8(5,0) & 10(6,2) \\ \quad 1(0,6) & 6(3,8) & 7(4,4) \\ \quad \text { Cavero } & 1(0,6) & 9(5,6) & 10(6,2) \\ \begin{array}{l}\text { Ayudante de } \\ \text { Matarife }\end{array} & 0(0,0) & 17(10,7) & 17(10,7) \\ \text { Mondonguero } & 0(0,0) & 13(8,2) & 13(8,2) \\ \text { Carnicero } & 0(0,0) & 8(5,0) & 8(5,0) \\ \text { Otros** } & 0(0,0) & 21(13,2) & 21(13,2)\end{array}$

\section{Ocupación previa***}

\begin{tabular}{crrr} 
Matarife & $4(2,5)$ & $30(18,8)$ & $34(21,4)$ \\
No refiere & $2(1,2)$ & $45(28,3)$ & $47(29,6)$ \\
Carnicero & $1(0,6)$ & $7(4,4)$ & $8(5,0)$ \\
Veterinario & $1(0,6)$ & $6(3,7)$ & $7(4,3)$ \\
Otros (mecánico) & $1(0,6)$ & $55(34,5)$ & $56(35,1)$ \\
Ordeñador & $0(0,0)$ & $7(4,4)$ & $7(4,4)$ \\
Total & $9(5,6)$ & $150(94,4)$ & $159(100,0)$ \\
\hline
\end{tabular}

${ }^{*} \mathrm{j}^{2}=0,026: 1^{\circ} \mathrm{L} ; \mathrm{P}=0,589 ;$ **Otros: caporal, ordeñador, becerrero, sabanero; $* * \star \mathrm{j}^{2}=6,281: 1^{\circ} \mathrm{L} ; \mathrm{P}=0,616$

antecedente de haber trabajado como matarifes y de manipular placentas, que es uno de los tejidos por los que estas bacterias tienen mayor afinidad $[12,27]$.

En la TABLA III se muestran otros factores de riesgo de los trabajadores, siendo frecuente el consumo de derivados lácteos sin pasteurizar $(95,6 \%)$, la manipulación de placenta $(45,3 \%)$ y el desconocimiento de los riesgos de su ocupación (35,8 \%). Además, el 95,6 \% de los trabajadores consumían derivados lácteos sin pasteurizar, y se ha descrito que éste es otro factor de riesgo para adquirir la infección por Brucella spp. [27, 30]. Otro factor de riesgo importante fue, que los trabajadores no utilizaban las medidas de protección (ni guantes, ni protectores nasales). La forma de transmisión aérea de la brucelosis es importante [15, $24,25,27] \mathrm{y}$, el riesgo de laceraciones representa una puerta de entrada para las bacterias [15, 16, 24].

Posiblemente, este comportamiento se debe al desconocimiento de los trabajadores sobre los diferentes mecanismos de trasmisión de la brucelosis. A pesar de que la mayoría de los trabajadores estaban expuestos a múltiples factores de riesgo para contraer

Tabla III

Factores de riesgo ocupacionales y manifestaciones clínicas en trabajadores de los mataderos del Edo. Bolívar y Soledad municipio Independencia (Edo. Anzoátegui)

\begin{tabular}{ccc}
\hline & $\begin{array}{c}\text { Presencia de } \\
\text { anticuerpos } \\
\text { contra }\end{array}$ & $\begin{array}{c}\text { Ausencia de } \\
\text { anticuerpos } \\
\text { contra }\end{array}$ \\
& Brucella spp. (\%) & Brucella spp. (\%) \\
\hline
\end{tabular}

\section{Factores de riesgos*}

Consumo de derivados

lácteos sin pasteurizar

$152(95,6)$

$7(4,4)$

Manipulación

de placenta

Conocimientos sobre

riesgos del trabajo

Conocimiento

sobre brucelosis

Uso de guantes y

protectores de nariz

Manifestaciones

clínicas**

Artralgia

$72(45,3)$

$87(54,7)$

$57(35,8)$

$102(64,2)$

$43(27,0)$

$116(73,0)$

$7(4,4)$

$152(95,6)$

Asintomáticos

$5(3,1)$

$59(37,1)$

$3(1,8)$

$60(37,7)$

Cefalea

$3(1,8)$

$41(25,7)$

Fiebre

$2(1,2)$

$27(16,9)$

Sudoración nocturna

$2(1,2)$

$33(20,8)$

Pérdida de apetito (anorexia)

* $\mathrm{j}^{2}=9,481: 1^{\circ} \mathrm{L} ; \mathrm{P}=0,512 ; * \star \mathrm{j} \mathrm{i}^{2}=5,154: 1^{\circ} \mathrm{L} ; \mathrm{P}=0,382$ 
brucelosis, esta seroprevalencia no deja de ser importante ya que se trata de una enfermedad con implicaciones en la salud humana y en la economía del país.

Las manifestaciones clínicas referidas por los trabajadores fueron: artralgias $(n=64 ; 40,3 \%)$, cefalea $(n=44 ; 27,7 \%)$ y sudoración nocturna $(n=35 ; 22,0 \%)$. Las artralgias predominaron en los pacientes seropositivos a Brucella spp. $(n=5 ; 3,1 \%)$. No se demostró asociación estadísticamente significativa entre las manifestaciones clínicas encontradas y la seropositividad a Brucella spp. $(P>0,05)$.

Los principales signos y síntomas que manifestaron los trabajadores eran inespecíficos, resultados similares ha sido evidenciados por otros autores [2, 6, 8].

De las 303 muestras de sueros obtenidas de los bovinos, se obtuvo una prevalencia de infección IgM por Brucella spp. del $11,2 \%(n=34)$. La mayor seropositividad se obtuvo en los mataderos ubicados en El Palmar, Upata y Soledad $(n=9 ; 2,9 \%$ en cada uno), seguido de San Félix y Guasipati ( $n=3 ; 0,9 \%$, respectivamente) y Tumeremo $(n=1 ; 0,3 \%)$. En el matadero ubicado en El Callao no se detectaron anticuerpos en los bovinos.

En el caso de los bovinos, la seroprevalencia de infección por Brucella spp. fue elevada (11,2\%); similar a la descrita en caprinos en el país $[9,20]$. En otras regiones de Venezuela se han señalado cifras elevadas de prevalencia de infección por Brucella spp., en Lara (13,1\%) [23] y en el estado Zulia (20,3 \%) [10]; así como también valores inferiores a la señalada en este estudio: $9 \%$ en Barinas y 2,9 \% en Guárico [31, 33].

En otras regiones de Latinoamérica se han señalado prevalencias superiores de brucelosis bovina, por ejemplo, en Colombia entre 1,7 a 32,2 \% [1], $0 \%$ en México [4], en la provincia de Parinacochas, Ayacucho, Perú, está ausente y en el caso de existir tendría una prevalencia inferior a 4,7 \% [34] y en Chile un $5 \%$ [13].

En otras latitudes se registran seroprevalencias de brucelosis inferiores a la demostrada en esta investigación, como por ejemplo en Egipto $(0,79 \%)$ [18]. En el sur de África, la prevalencia fue $11,0 \%$ (22 de 200), similar a la demostrada en este estudio, empleando otra técnica diagnóstica; aunque cuando se utilizó la prueba de ELISA en la misma población de bovinos, la prevalencia fue mucho menor 5,5\% (11 de 200) [21].

La seroprevalencia demostrada en bovinos, tiene especial importancia ya que representa un riesgo ocupacional, donde están expuestos, tanto el personal que labora en los mataderos como la población en general.

La seroprevalencia de brucelosis se corrobora con los informes que muestran una tasa media positiva del 10,5 \% y valores aún más altos en algunas zonas del país [9, 10, 14].

Se recomienda realizar pruebas de serologías frecuentes, vigilancia continua, la implementación de medidas preventivas que eviten la entrada del patógeno al rebaño, así como aplicación de vacunas, las cuales podrían ser efectivas en el control de la incidencia de la infección por Brucella spp.

Las autoridades de salud pública deben educar a los agricultores, ganaderos y al público en general con respecto a la prevención de la enfermedad, con especial hincapié a las personas con riesgo ocupacional y en especial aquellas que trabajan en los mataderos. Como la brucelosis es clínicamente inespecífica, debería incluirse sistemáticamente en el diagnóstico etiológico de las enfermedades infecciosas en estas poblaciones en riesgo.

\section{CONCLUSIONES}

La mayoría de los trabajadores de los mataderos evaluados del estado Bolívar y de Soledad, municipio Independencia, estado Anzoátegui, están expuestos a múltiples factores de riesgo para contraer brucelosis; sin embargo, la seroprevalencia de brucelosis en ellos fue baja (5,6 \%), aunque elevada entre los bovinos (11,2\%). Estos hallazgos confirman que la infección por Brucella spp., circula entre el ganado vacuno que se consume y demuestra un riesgo para los trabajadores en los mataderos seleccionados y para los consumidores de carne de res o lácteos, cocidos de manera inadecuada o no pasteurizados, siendo necesarios programas de control.

\section{REFERENCIAS BIBLIOGRÁFICAS}

[1] ANDRADE-BECERRA, R.J.; VARGAS, J.C. Seroprevalencia de Brucelosis bovina en el Altiplano Boyacense, ColombiaSuramérica. Rev. Científ. FCV-LUZ. XXIX (3): 192-197. 2019.

[2] APPANNANAVAR, S.B.; SHARMA, K.; VERMA, S.; SHARMA, M. Seroprevalence of Brucellosis: a 10-year experience at a tertiary care center in north India. Indian J. Pathol. Microbiol. 55:271-272. 2012.

[3] ASAMBLEA GENERAL UNESCO 59 ${ }^{\mathrm{TH}}$., SEUL, COREA. Declaración de Helsinki de laAsociación Médica Mundial. Principios éticos para las investigaciones médicas en seres humanos, octubre 2008. En línea: https://bit.ly/3gp4nmQ. 01.12.2015.

[4] BARAJAS, J. Aplicación de la técnica inmunoenzimática de Elisa para estudios epidemiológicos de enfermedades de ganado bovino en el trópico de México. Rev. Cien. Vet. 8: 85-151. 1998.

[5] Bello, A.; MARÍN, C.; MOGOLLÓN, P.; HERRERA, M.; PACHECHO, S.; RODRÍGUEZ, M.; GIL, R.; DE LASERNA, R.; DE SALMERON, C. Estudios epidemiológicos sobre brucelosis humana suina, canina y humana en establecimientos agrícolas de Punta Palmita (Venezuela) 1975. Vet. Trop. 3:3 - 14. 1978.

[6] BONFOH, B.; KASYMBEKOV, J.; DÜRR, S.; TOKTOBAEV, N.; DOHERR, M.G.; SCHUETH, T.; ZINSSTAG, J.; SCHELLING, E. Representative seroprevalences of brucellosis in humans and livestock in Kyrgyzstan. EcoHealth. 9:132-138. 2012.

[7] CAMPOS, V.M.; PÉREZ, M.; CÓRDOVA, A. Evaluation of the Economic Losses of Events Associated With Reproductive Failure in Female Bovine Brucellosis and Dairy Farms of the Basin of Tizayuca, Hidalgo, Mexico. Rev. Científ. FCV-LUZ. XX (2): 190-195. 2010.

[8] CERVERA-HERNÁNDEZ, M.E.; ORDAZ-VÁZQUEZ, A.; TORRES-GONZÁLEZ, P.; CHÁVEZ-MAZARI, B.; SOBERANISRAMOS, O.; SIFUENTES-OSORNIO, J.; PONCE DE LEÓN, A.; BOBADILLA DEL V., M. Seroprevalence of brucellosis among dairy farm workers in Mexico. Sal. Pub. Mex. 58:36670. 2016.

[9] DE LORD, V.; NIETO, S.; SANDOVAL, E.; MELÉNDEZ, G.; RUIZ, R. Brucelosis en caprinos: Estudios serológicos y bacteriológicos en Venezuela. Vet. Trop. 12: 27-37. 1987. 
[10] D`POOL, G.; RIVERA, S.; TORRES, T.; PÉREZ, M.; GARCÍA, A.; CASTEJÓN, O.; ROJAS, N. Prevalencia de brucelosis bovina mediante ELISA competitivo en el Municipio de Cañada de Urdaneta, estado Zulia, Venezuela. Rev. Científ. FCV-LUZ. XIV (2): 168-176. 2004.

[11] DRG INTERNATIONAL, INC. USA. DRG® Brucella IgM ELISA (EIA-3456). 2011. En línea: https://bit.ly/3wBtTKY. 15.10.2006

[12] ESMAEILI, S.; BAGHERI-AMIRI, F.; MOKHAYERI, H.; KAYEDI, M.H.; MAURIN, M.; ROHANI, M.; MOSTAFAVI, E. Seroepidemiological study of $Q$ fever, brucellosis and tularemia in butchers and slaughterhouses workers in Lorestan, western of Iran. Comp. Immunol. Microbiol. Infect. Dis. 66:101-322. 2019.

[13] FELMER, R.; ZUÑIGA, J.; LÓPEZ, A.; MIRANDA, H. Prevalencia y distribución espacial de brucelosis, leucosis bovina, diarrea viral bovina y rinotraqueitis infecciosa bovina a partir del análisis ELISA de estanques prediales en lecherías de la IX Región, Chile. Arch. Med. Vet. 41:17-26. 2009.

[14] FRANCISCO, J.; VARGAS, O. Brucellosis in Venezuela. Vet. Microbiol. 90: 39-44. 2002.

[15] FRANC, K.A.; KRECEK, R.C.; HÄSLER, B.N.; ARENASGAMBO, A. Brucellosis remains a neglected disease in the developing world: a call for interdisciplinary action. BMC Public Health. 18:1-9. 2018.

[16] GARCÍA, P.; CARREÑO, T.; ROdRÍGuEZ, R. Estudio descriptivo de la brucelosis en la provincia de Almería. Evolución de los mecanismos de transmisión. Med. Famil. 2: 46-52. 2001.

[17] GONÇALVES, D.D.; TELES, P.S.; DOS REIS, C.R.; LOPES, F.M.; FREIRE, R.L.; NAVARRO, I.T.; ALVES, L.A.; MULLER, E.E.; DE FREITAS, J.C. Seroepidemiology and occupational and environmental variables for leptospirosis, brucellosis and toxoplasmosis in slaughterhouse workers in the Paraná State, Brazil. Rev. Inst. Med. Trop. Sao Paulo. 48:135-140. 2006.

[18] HEGAZY, Y.M.; MOLINA-FLORES, B.; SHAFIK, H.; RIDLER, A.L.; GUITIAN, F.J. Ruminant brucellosis in Upper Egypt (2005-2008). Prev. Vet. Med. 101:173-181. 2011.

[19] JAMA'AYAH, M.Z.; HEU, J.Y.; NORAZAH, A. Seroprevalance of brucellosis among suspected cases in Malaysia. Malays. J. Pathol. 33: 31-34. 2011.

[20] JAVITT-JIMENEZ, M.J.; PÁEZ, Z.; DURAN, J.; MELÉNDEZ, I. Seroprevalencia de la brucelosis en pequeños rumiantes. Municipio Torres. Año 2008. REDVET. 2009. 10(8). En línea: https://bit.ly/3pRsVI9. 27.02.2019.

[21] KOLO, F.B.; ADESIYUN, A.A.; FASINA, F.O.; KATSANDE, C.T.; DOGONYARO, B.B.; POTTS, A.; MATLE, I.; GELAW, A.K.; VAN HEERDEN, $\mathrm{H}$. Seroprevalence and characterization of Brucella species in cattle slaughtered at Gauteng abattoirs, South Africa. Vet. Med. Sci. 5(4):545-555. 2019.

[22] LOPES, E.; MIGRANAS, R.; PÉREZ, A.; MAGOS, C.; SALVATIERRA, B.; TAPIA-CONVER, R.; VALDESPINO, J.L.; SEPÚLVEDA, J. Seroepidemiología de la brucelosis en México. Sal. Publ. Mex. 34: 230-240. 1992.

[23] MALDONADO, J.; KOWALSKI, A. MILLA, M.; RODRÍGUEZ, M.; VILLASMIL, C. Implementación de la prueba del anillo en leche y ELISA indirecto para el diagnóstico de Brucelosis en rebaños de doble propósito en el estado Lara, Venezuela. Rev. Científ. FCV-LUZ. XX (3):240-244. 2010.

[24] MUKHTAR, F. Brucellosis in a high risk occupational group: seroprevalence and analysis of risk factors. J. Pak. Med. Assoc. 60:1031-1034. 2010.

[25] NJERU, J.; WARETH, G.; MELZER, F.; HENNING, K.; PLETZ, M.W.; HELLER, R.; NEUBAUER, H. Systematic review of brucellosis in Kenya: disease frequency in humans and animals and risk factors for human infection. BMC Public Health. 16(1):1-15. 2016.

[26] PAPPAS, G.; PAPADIMITRIOU, P.; AKRITIDIS, N.; CHRISTOU, L.; TSIANOS, E.V. The new global map of human brucellosis. Lancet Infect. Dis. 6: 91-99. 2006.

[27] RAHMAN, A.K.; DIRK, B.; FRETIN, D.; SAEGERMAN, C.; AHMED, M.U.; MUHAMMAD, N.; HOSSAIN, A.; ABATIH, E. Seroprevalence and risk factors for brucellosis in a high-risk group of individuals in Bangladesh. Foodborne Pathog. Dis. 9:190-197. 2012.

[28] RIVERA, S.; CURIEL, J.; ROJAS, N.; SANGRONI, I.; URDANETA, N. Epidemiología serológica de la Brucelosis Bovina en el Municipio Rosario de Perijá (Zulia. Venezuela). Rev. Científ. FCV-LUZ. V (2): 117-124. 1995.

[29] SERRA, J.; GODOY, P. Incidencia, etiología y epidemiología de la brucelosis en un área rural de la provincia de Lleida. Rev. Esp. Sal. Publ. 74: 45-53. 2000.

[30] SHIMOL, S.B.; DUKHAN, L.; BELMAKER, I.; BARDENSTEIN, S.; SIBIRSKY, D.; BARRETT, C.; GREENBERG, D. Human brucellosis outbreak acquired through camel milk ingestion in southern Israel. Isr. Med. Assoc. J. 14: 475-478. 2012.

[31] SOTO-REVEROL, N. Seroprevalencia de la Brucelosis y su impacto reproductivo en un Rebaño Brahman. 2013. Rev. Col. Med. Vet. Edo. Lara. 3:5(1). En Línea: https://bit. ly/3wqSwdx.1.04.2020

[32] SVANOVA BIOTECH, AB. Brucella - Ab competitive C-ELISA. SVANOVIR ${ }^{\circ}$. ELISA test for the detection of Brucella antibodies in serum. 2006. Discriminating between infected and vaccinated animals. En Línea: https://bit.ly/3iGxkvZ. 1.07.2015

[33] TAMASAUKAS, R.; PURROY, R.; RODRÍGUEZ, H.; RUIZ, I.; ROA, N.; LABRADOR, C. Seroprevalencia de tripanosomiasis y brucelosis bovina en fincas integradas a la producción de maíz, de la zona alta de los Municipios Roscio y Ortiz, Edo. Guárico, Venezuela. Rev. Científ. FCV-LUZ. XII (Supl 2): 630-634. 2002.

[34] VALDIVIA, L.; RIVERA, H. Seroprevalencia de Brucella spp. en bovinos criollos de crianza extensiva de la provincia de Parinacochas, Ayacucho. Rev. Inv. Perú. 14: 174-177. 2003.

[35] VARGAS, F. Situación epidemiológica de la brucelosis en Venezuela. Gac. Cien. Vet. 8: 1-15. 2003.

[36] WHATMORE, A.M.; KOYLASS, M.S.; MUCHOWSKI, J.; EDWARDS-SMALLBONE, J.; GOPAUL, K.K.; PERRETT, L.L. Extended multilocus sequence analysis to describe the global population structure of the genus Brucella: phylogeography and relationship to biovars. Front. Microbiol. 7:2049. 2016. 